\title{
Dairy management practices associated with multi-drug resistant fecal commensals and Salmonella in cull cows: a machine learning approach
}

\author{
Pranav S Pandit ${ }^{1}$, Deniece R. Williams ${ }^{2}$, Paul Rossitto ${ }^{2}$, John M Adaska ${ }^{3}$, Richard Pereira ${ }^{4}$, Terry W. Lehenbauer \\ ${ }^{2,4}$, Barbara A. Byrne ${ }^{5}$, Xunde Li ${ }^{6}$, Edward R Atwill ${ }^{6}$, Sharif S. Aly ${ }^{\text {Corresp. } 2,4}$ \\ ${ }^{1}$ EpiCenter for Disease Dynamics, One Health Institute, School of Veterinary Medicine, University of California Davis, Davis, California, The United States \\ of America \\ ${ }^{2}$ Veterinary Medicine Teaching and Research Center, University of California, Davis, Tulare, California, The United States of America \\ 3 California Animal Health and Food Safety Laboratory System, University of California, Davis, Tulare, California, The United States of America \\ 4 Department of Population Health and Reproduction, School of Veterinary Medicine, University of California, Davis, Davis, California, The United States of \\ America \\ 5 Department of Pathology, Microbiology \& Immunology, School of Veterinary Medicine, University of California, Davis, California, The United States of \\ America \\ ${ }^{6}$ Western Institute for Food Safety and Security, University of California, Davis, Davis, California, The United States of America \\ Corresponding Author: Sharif S. Aly \\ Email address: saly@ucdavis.edu
}

Background: Understanding the effects of herd management practices on the prevalence of multidrug-resistant pathogenic Salmonella and commensals Enterococcus spp. and Escherichia coli in dairy cattle is key in reducing antibacterial resistant infections in humans originating from food animals. Our objective was to explore the herd and cow level features associated with the multi-drug resistant, and resistance phenotype shared between Salmonella, E. coli, and Enterococcus spp. using machine learning algorithms. Methods: Randomly collected fecal samples from cull dairy cows from six dairy farms in central California were tested for multi-drug resistance phenotypes of Salmonella, E. coli, and Enterococcus spp. Using data on herd management practices collected from a questionnaire, we built three machine learning algorithms (decision tree classifier, random forest, and gradient boosting decision trees) to predict the cows shedding multidrugresistant Salmonella and commensal bacteria. Results: The decision tree classifier identified rolling herd average milk production as an important feature for predicting fecal shedding of multi-drug resistance in Salmonella or commensal bacteria. The number of culled animals, monthly culling frequency and percentage, herd size, and proportion of Holstein cows in the herd were found to be influential herd characteristics predicting fecal shedding of multidrug-resistant phenotypes based on random forest models for Salmonella and commensal bacteria. Gradient boosting models showed that higher culling frequency and monthly culling percentages were associated with fecal shedding of multidrug 
resistant Salmonella or commensal bacteria. In contrast, an overall increase in the number of culled animals on a culling day showed a negative trend with classifying a cow as shedding multidrug-resistant bacteria. Increasing rolling herd average milk production and spring season were positively associated with fecal shedding of multidrug- resistant Salmonella. Only 6 individual cows were detected sharing tetracycline resistance phenotypes between Salmonella and either of the commensal bacteria. Discussion: Percent culled and culling rate reflect the increase in culling over time adjusting for herd size and were associated with shedding multidrug resistant bacteria. In contrast, number culled was negatively associated with shedding multidrug resistant bacteria which may reflect producer decisions to prioritize the culling of otherwise healthy but low-producing cows based on milk or beef prices (with respect to dairy beef), amongst other factors. Using a data-driven suite of machine learning algorithms we identified generalizable and distant associations between antimicrobial resistance in Salmonella and fecal commensal bacteria, that can help develop a producer-friendly and data-informed risk assessment tool to reduce shedding of multidrug-resistant bacteria in cull dairy cows. 


\section{Dairy management practices associated with multi-}

\section{2 drug resistant fecal commensals and Salmonella in}

\section{3 cull cows: a machine learning approach}

4 Pranav S. Pandit ${ }^{1}$, Deniece R. Williams ${ }^{2}$, Paul Rossitto ${ }^{2}$, John Adaska ${ }^{3}$, Richard Pereira ${ }^{4}$, Terry

5 W. Lehenbauer ${ }^{2,4}$, Barbara A. Byrne ${ }^{5}$, Xunde Li ${ }^{6}$, Robert Atwill ${ }^{6}$, Sharif S. Aly ${ }^{2,4 *}$

6

$7 \quad{ }^{1}$ EpiCenter for Disease Dynamics, School of Veterinary Medicine, University of California

8 Davis, Davis, CA, USA

${ }^{2}$ Veterinary Medicine Teaching and Research Center, University of California, Davis, Tulare, CA, USA

${ }^{3}$ California Animal Health and Food Safety Laboratory System, University of California, Davis, Tulare, CA, USA

${ }^{4}$ Department of Population Health and Reproduction, School of Veterinary Medicine, University of California, Davis, Davis, CA, USA

${ }^{5}$ Department of Pathology, Microbiology \& Immunology, School of Veterinary Medicine, University of California, Davis, California

${ }^{6}$ Western Institute for Food Safety and Security, University of California, Davis, Davis, CA, USA

*Corresponding Author: Sharif Aly; email address: saly@ucdavis.edu 


\section{Abstract}

26 Background: Understanding the effects of herd management practices on the prevalence of 27 multidrug-resistant pathogenic Salmonella and commensals Enterococcus spp. and Escherichia

28 coli in dairy cattle is key in reducing antibacterial resistant infections in humans originating from

29 food animals. Our objective was to explore the herd and cow level features associated with the

30 multi-drug resistant, and resistance phenotype shared between Salmonella, E. coli, and

31 Enterococcus spp. using machine learning algorithms.

32 Methods: Randomly collected fecal samples from cull dairy cows from six dairy farms in central

33 California were tested for multi-drug resistance phenotypes of Salmonella, E. coli, and

34 Enterococcus spp. Using data on herd management practices collected from a questionnaire, we

35 built three machine learning algorithms (decision tree classifier, random forest, and gradient

36 boosting decision trees) to predict the cows shedding multidrug-resistant Salmonella and

37 commensal bacteria.

38 Results: The decision tree classifier identified rolling herd average milk production as an 39 important feature for predicting fecal shedding of multi-drug resistance in Salmonella or 40 commensal bacteria. The number of culled animals, monthly culling frequency and percentage, 41 herd size, and proportion of Holstein cows in the herd were found to be influential herd 42 characteristics predicting fecal shedding of multidrug-resistant phenotypes based on random 43 forest models for Salmonella and commensal bacteria. Gradient boosting models showed that 44 higher culling frequency and monthly culling percentages were associated with fecal shedding of 45 multidrug resistant Salmonella or commensal bacteria. In contrast, an overall increase in the 46 number of culled animals on a culling day showed a negative trend with classifying a cow as 47 shedding multidrug-resistant bacteria. Increasing rolling herd average milk production and spring 
48 season were positively associated with fecal shedding of multidrug-resistant Salmonella. Only 6

49 individual cows were detected sharing tetracycline resistance phenotypes between Salmonella

50 and either of the commensal bacteria.

51 Discussion: Percent culled and culling rate reflect the increase in culling over time adjusting for

52 herd size and were associated with shedding multidrug resistant bacteria. In contrast, number

53 culled was negatively associated with shedding multidrug resistant bacteria which may reflect

54 producer decisions to prioritize the culling of otherwise healthy but low-producing cows based

55 on milk or beef prices (with respect to dairy beef), amongst other factors. Using a data-driven

56 suite of machine learning algorithms we identified generalizable and distant associations

57 between antimicrobial resistance in Salmonella and fecal commensal bacteria, that can help

58 develop a producer-friendly and data-informed risk assessment tool to reduce shedding of

59 multidrug-resistant bacteria in cull dairy cows. 


\section{Introduction}

61 The Centers for Disease Control and Prevention (CDC) estimates that more than 2.8 million

62 antimicrobial resistant infections occur in the U.S. with more than 35,000 deaths annually

63 (Control \& Prevention 2019). Amongst the resistant bacteria, the CDC classifies nontyphoidal

64 Salmonella enterica as a serious public health threat (Control \& Prevention 2019). Salmonella is

65 an important foodborne zoonotic agent in the U.S. (Scallan et al. 2011) and several studies

66 reported on its prevalence in cull cattle. Troutt et al found that the prevalence of Salmonella in

67 cecal contents of dairy cattle at slaughter in the Western U.S. ranged between $9.6 \%$ and $35.6 \%$ in

68 the winter, and between $32.3 \%$ and $93 \%$ in the summer (Troutt et al. 2001). More recent studies

69 reported on the associations between herd management and seasonal differences on the

70 prevalence of Salmonella in fecal samples of cull dairy cows collected quarterly from seven

71 California dairies with an overall Salmonella shedding prevalence of $3.42 \%(95 \%$ CI $1.28,5.56)$

72 (Abu Aboud et al. 2016). Pereira et al. (Pereira et al. 2019) followed six of the same California

73 dairies for a second year showing an increase in Salmonella shedding prevalence $(30.6 \%$; 95\%

74 CI 262-350). The increase in the prevalence was speculated to be due to increased rainfall and

75 drier summer season and herd changes that occurred during the latter study (Adaska et al. 2020).

76 The latter study explored how the study herd and cow level features were associated with

77 shedding of antimicrobial resistant Salmonella and although $60 \%$ of the isolates were pan-

78 susceptible, the remaining isolates were found to be resistant to different medically important

79 antimicrobial drugs (MIAD) defined as antimicrobial drugs (AMD) that are important for

80 therapeutic use in humans, with $12 \%$ of the isolates being multidrug resistance to more than two 81 drug classes. 
82 Fecal commensals such as E. coli and Enterococcus spp. can acquire mobile gene elements that

83 encode antimicrobial resistance to these species (Aidara-Kane et al. 2018). Given the

84 documented antimicrobial resistance in Salmonella isolated from cull dairy cows, further

85 research on the similarity between resistance patterns of fecal commensals and Salmonella shed

86 in feces of dairy cattle is needed.

87 Traditional risk factor approaches (mixed-effects modeling) can often have limitations

88 due to high-dimensional, imbalanced, and non-linear data and can perform poorly in cases of

89 large number predictive variables. To overcome these, we used a suite of classification tree

90 models, a group of supervised machine learning models that can handle various types of data and

91 handle interactions between predictive variables. Classification tree models like random forest,

92 gradient boosting have been found useful in investigating the prevalence and associated risk

93 factors of bovine viral diarrhea virus (Machado et al. 2015), swine pneumonia (Mollenhorst et al.

94 2019), and mastitis in dairy cattle (Hyde et al. 2020). In the study reported here, our objective

95 was to explore the herd and cow level features associated with the multi-drug resistance and

96 resistance phenotype shared between Salmonella, E. coli, or Enterococcus spp. using machine

97 learning algorithms.

98 Materials \& Methods

100 Farms surveys and sampling

101 The study was approved by the University of California, Davis's Institutional Animal

102 Care and Use Committee (protocol number 18019). Six dairy farms located in the San Joaquin

103 Valley of California were reenrolled as a convenience sample and followed up for a second year

104 after being part of an earlier study (Abu Aboud et al. 2016). Briefly, cull cows were identified for 
105 fecal sampling once during each season between 2015 and 2016, specifically during summer

106 (July 1-September 30, 2015), fall (October 1-December 31, 2015), winter (January 1-March 31,

107 2016) and spring (April 1-June 30,2016). The choice of sampling week to collect fecal samples

108 from the cull cows during any of the four seasons was also by convenience. From the list of cows

109 selected by the dairy farms for culling, 10 cows were randomly selected for fecal sampling on

110 the day of their removal from the herd using a random number generator (Excel; Microsoft

111 Corp., Redmond, WA, USA). Several lists of random numbers were prepared in multiples of 10

112 ranging from 11-20 to 91-100 cows and the respective list was selected depending on the

113 number of cows presented for culling on the day of sampling. If a producer had less than 11

114 cows presented for culling, all the cows were sampled. An individual disposable sleeve was used

115 to manually collect fecal samples from the rectum of each selected cow. The fecal samples were

116 transported to the Aly Lab (Dairy Epi Lab) on wet ice for processing on the same day.

117 A survey was also completed with the help of the herd manager on the same sampling

118 day. The survey questions were described in an earlier report (Pereira et al., 2019). Briefly, the

119 questions targeted management of the herd in the previous 4 months and collected information

120 on herd size, breed, rolling herd average, cull rate, frequency of culling per month, the proportion

121 of cows sold for beef (compared to as dairy), proportion and reasons for condemnation of culled

122 cows. The survey also collected information on the proportion of culled cows that received

123 injectable medical treatments in the 3 weeks prior to culling, the role of dairy staff allowed to

124 treat cows on the dairy, practices to avoid drug residue violations (use of specific drug types,

125 following withdrawal times, milk and/or urine testing prior to the cow being culling, or other

126 practices), tracking of drug withdrawal intervals, having a drug inventory system in place, and

127 extra-label drug use (ELDU, frequency and familiarity). Finally, a backup of the herd record 
128 software was obtained to collect information on the culled cows' milk production and health

129 events. A relational database was used to house and merge data from the surveys, dairy records, 130 and test results (Access; Microsoft Corp., Redmond, WA, USA).

\section{Bacteriological culture}

The California Animal Health and Food Safety (CAHFS) laboratory conducted all the study sample testing for Salmonella as described by Adaska et al. (Adaska et al. 2020). Briefly, 1 gram of feces was inoculated into tetrathionate selective enrichment broth and incubated at 37

$\pm 2{ }^{\circ} \mathrm{C}$. The next morning a cotton swab was used to inoculate the overnight broth onto XLD and

XLT-4 plates and these were incubated overnight at $37 \pm 2{ }^{\circ} \mathrm{C} . \mathrm{H}_{2} \mathrm{~S}$ positive, Salmonella suspect

colonies from each set of plates were subcultured onto individual bi-plates (5\% sheep blood agar-MacConkey agar) and incubated overnight at $37 \pm 2{ }^{\circ} \mathrm{C}$. One colony from each bi-plate was used for biochemical testing which included triple sugar iron, urea, motility indole ornithine, citrate, O-nitrophenyl-beta-D-galactopyranoside, and lysine iron agar slants. Serogrouping and serotyping were performed, using the White-Kauffmann-Le Minor scheme (Grimont \& Weill 2007) on colonies with biochemical test results consistent with Salmonella (Quinn et al. 2002)

145 previously (Li et al. 2014). Briefly, a $40 \mathrm{~mL}$ solution of buffered peptone water containing $5 \mathrm{~g}$ of

146 feces in a $50 \mathrm{~mL}$ polypropylene tube is homogenized using a mechanical shaker for $15 \mathrm{~min}$

147 before filtering using gauze. 1000,100 , and $10 \mu \mathrm{l}$ of the filtered solution were then streaked on

148 CHROMAgar ECC (Chromagar, Paris) and Enterococcus Indoxyl- $\beta$-D-Glucoside agar (mEI)

149 plates (Becton, Dickinson and Company, Franklin Lakes, NJ) both incubated at $37^{\circ} \mathrm{C}$ for $24 \mathrm{~h}$.

150 Reference strains ATCC 25922 (E. coli) and ATCC 29212 (Enterococcus faecalis) were plated 
151 on agar plates as positive controls. Two pure colonies were isolated of each species after

152 presumptive colonies were confirmed using biochemical tests (E. coli were confirmed using

153 urea, indole, triple sugar iron, Methyl Red-Voges-Proskauer and Simmons citrate; Enterococcus

154 were confirmed using bile esculin, brain heart infusion agar, and growth in broth with and 155 without $6.5 \% \mathrm{NaCl})$.

157 Antimicrobial susceptibility testing

158 Salmonella and E. coli bacterial resistance was evaluated with a broth microdilution 159 method using a Gram-negative Sensititre plate (CMV2AGNF) and Enterococcus spp. evaluated 160 on gram-positive Sensititre plate (CMV3AGPF) (Sensititre, Thermo Fisher Scientific, MA, 161 USA) according to the manufacturer's instructions and as described in previous studies (Li et al. 162 2018; Pereira et al. 2019). The minimum inhibitory concentration (MIC) values were the lowest 163 concentrations of AMD that inhibited visible growth of bacteria. Interpretations of antimicrobial 164 resistance were based on breakpoints recommended by the National Antimicrobial Resistance 165 Monitoring System (https://www.cdc.gov/narms/antibiotics-tested.html; and 166 https:/www.ars.usda.gov/ARSUserFiles/60400520/NARMS/ABXEntero.pdf) and the Clinical 167 and Laboratory Standards Institute (CLSI 2014; CLSI 2018). Due to the inherent resistance of

168 Salmonella to cephalosporins, aminoglycosides, lincosamides, oxazolidinones, and 169 glycopeptides were excluded from the antimicrobial resistance analysis. In addition, the 170 following drug classes to which $E$. coli is inherently resistant were excluded from the analysis:

171 lincosamides, oxazolidinones, penicillins, streptogramins, glycopeptides. Similarly, the drug 172 classes exclude due to the inherent resistance of Enterococcus spp. included cephalosporins,

173 lincosamides, fluoroquinolones, aminoglycosides, aminocyclitols, sulfonamides, folate pathway 
174 antagonists. Isolates from any of the three species (Salmonella, E. coli, Enterococcus spp.) were

175 identified as multi-drug resistant if resistance to at least one AMD in each of three or more drug

176 classes was observed (Magiorakos et al., 2012).

\section{Development of classification algorithms}

178 For cows that were found positive for Salmonella, classification tree models were

179 developed to test if herd management practices and features related to dairy cows can predict

180 multi-drug resistance phenotype in Salmonella and fecal commensal E. coli, and Enterococcus

181 spp. isolated from the same cows. A cow was considered to shed MDR bacteria if any of its

182 Salmonella, Enterococcus spp. and/or E. coli isolates showed resistance for three or more

183 antimicrobial classes (regardless of whether the species with resistance was Salmonella,

184 Enterococcus spp. and/or E. coli), in which case the cow was labeled as 'shedding bacteria with

185 multi-drug resistance (MDR) phenotype' (numerical label =2). In contrast, if a cow shed

186 bacteria that were resistant to only one or two antimicrobial classes (regardless of species) the

187 bacteria isolated were labeled as 'shedding bacteria with antimicrobial resistance (AMR)

188 phenotype' (numerical label $=1$ ). If the bacteria isolated from a cow did not show any resistance

189 across all three bacterial species to any AMD, the bacteria were labeled as non-resistant

190 (numerical label $=0$ ). The mutually exclusive definitions were necessary to develop a single

191 model that predicts one of three resistance states MDR, AMR, or no resistance. Similarly, cows

192 were also classified based on resistance phenotypes separately observed in each bacterial species

193 isolated (three separate classification labels based on resistance phenotypes of Salmonella,

194 Enterococcus spp. and E. coli isolates). Finally, a classification was generated based on resistant

195 phenotypes observed in either commensal bacteria (Enterococcus spp. and E. coli) shed in feces 
196 collectively. Using features from herd surveys, classification tree models were trained to predict

197 the MDR phenotype of bacteria shed in the feces of the study cows.

198 Three machine learning algorithms, specifically decision tree classifier (DTC), random

199 forest (RF), and gradient boosting (GB) were developed to explore the risk factors for resistance

200 phenotypes in the study isolates using herd survey data, specifically to predict the multilabel

201 outcome based on the resistance phenotype of bacteria shed by cows (non-resistant, AMR, and

202 MDR). For each algorithm, data from the entire study cohort described by Pereira et al. (238

203 cows) were used, except models specific to predicting AMR phenotypes in Salmonella were

204 restricted to the cohort of 58 Salmonella positive cows. Table 1 describes all classification

205 algorithms trained and developed for various bacteria-specific outcomes.

206

207

For all three classification algorithms, 25 features related to herd management and 12

208 features related to individual cows collected from the survey were used as predictive features

209 (Table 2). The DTC generates an optimum tree based on attributes by recursive selections to split

210 data into classes and it was only used to visualize the optimum tree and as a contrast to the

211 remaining classification tree models (RF and GB) that prevent overfitting, unlike DTC. The RF

212 and GB algorithms both generate a series of recursive trees of binary splits for randomly sampled

213 predictor variables. While all tree classification algorithms handle interaction effects between

214 predictors, within GB, boosting builds and combines collective models improving the predictive

215 performance of many weak models substantially, and fits complex nonlinear relationships (Elith

216 et al. 2008). For the validation of each algorithm, the data was split into training and validation

217 datasets. To identify the best hyperparameters of the classification algorithms (hyper-tuning), a

218 grid search was implemented on the training dataset. Grid search is a tuning technique that 
219 computes optimum values of model parameters automatically by an exhaustive search performed 220 on a set of parameter values. Training datasets, composed of $80 \%$ of the data, were randomly

221 selected for gird search, maintaining the outcome proportional to the original dataset, with three-

222 fold cross-validation (internal validation). Model parameters tested for hyper-tuning of RF and

223 GB are given in Table 1. The best performing model parameters were chosen based on the

224 accuracy of the model. For each algorithm, the external validation of the best performing model 225 was done on the validation dataset (20\% remaining random sample of the original dataset) to 226 quantify the performance of the model on the completely independent validation dataset. The 227 DTC and RF models were implemented using the Scikit-learn machine learning package (Pedregosa et al. 2011) and GB was implanted using XGBoost in python (Chen \& Guestrin 229 2016).

Validated models were eventually fit on the complete dataset to produce the final 231 predictions. The relative influence (importance) of features for the random forest model was 232 estimated using average gini importance, permutation, and feature drop methods. The gini 233 importance for a feature is defined as the sum over the number of splits (across all tress) that 234 include the feature, proportionally to the number of samples it splits (Breiman 2001). The 235 gradient boosting model with the XGboost platform was evaluated using Shapely Additive 236 Explanations (SHAP) that assigned each predictive feature an additive feature unifying six 237 existing methods (Lundberg \& Lee 2017). Partial dependence of gradient boosted model 238 prediction on model features (expected output response trend as a function of feature) was 239 explored to understand the associations of the herd and cow-related features with predictions

240 (Friedman 2001). The Python code used for pre-processing the data, training and validating 
241 models and generating figures can be found here in the Zenodo repository,

242 https://doi.org/10.5281/zenodo.4387017.

243

244 Results

245

Salmonella isolates were detected in the feces of 58 cows $(24.4 \% \pm 2.8$ out of 238 cows $)$

246

on the six dairy herds. Two herds had no Salmonella positive samples throughout the study

247 period while for others the prevalence ranged from $12.5 \%( \pm 5.2, n=40)$ to $70.0 \%( \pm 7.2, n=40)$.

248

The most common reason reported for culling was low milk production $(65.1 \%, \pm 3.1)$

249 followed by poor reproduction $(31.1 \%, \pm 3.0)$. Lameness $(10.5 \%, \pm 1.9)$ and mastitis $(10.1 \%$,

$250 \pm 1.9)$ were the other reasons reported in the survey. Of the sampled cull cows, $15.5 \%( \pm 2.3)$

251 were reported as having received AMD as part of a treatment protocol for the condition resulting

252 in their culling decision. In contrast, 5.9\% $( \pm 1.5)$ received anti-inflammatory drugs for the

253 condition resulting in their culling decision.

254 Distribution of antimicrobial resistance

255 Predominant resistance phenotypes detected

256 The prevalence of AMR and MDR phenotypes in Salmonella was reported previously by

257 Pereira et al. (Pereira et al. 2019). Briefly, tetracyclines were the most prevalent drug class for

258 which Salmonella were resistant and $12 \%$ of the study isolates tested positive for MDR

259 Salmonella (Pereira et al. 2019). Within Enterococcus spp. isolates, the most common AMR

260 phenotype detected was for nitrofuran antimicrobials, $(10.83 \%, \pm 2.47)$. The most common type

261 of MDR phenotype in Enterococcus spp. isolates was resistance to oxazolidinones, nitrofuran

262 antibacterials, and macrolides $(5.73 \%, \pm 1.85)$. Frequencies of all resistance phenotypes observed

263 in Enterococcus spp. are presented in Table 3.

Peer] reviewing PDF | (2021:01:57027:2:0:NEW 24 May 2021) 
MDR phenotype was detected more than once (aminoglycosides, tetracyclines, amphenicols,

$2.47 \%, \pm 1.72$ ). Frequencies of all resistance phenotypes observed in E. coli are presented in

268 Table 4.

\section{Seasonality of multi-drug resistance}

The overall prevalence of shedding MDR bacteria (Salmonella, E. coli, and Enterococcus

271 spp.) in cows was $30.54 \%,( \pm 2.97, \mathrm{n}=238)$. The prevalence of cows shedding bacteria that are

272 resistant to $<=2$ antimicrobial drug classes $(A M R)$ was $43.93 \%( \pm 3.21, n=238)$. The highest

273 prevalence of MDR was seen in the summer $(50.00 \% \pm 11.18)$ followed by fall $(34.00 \%, \pm 4.73)$.

274 The highest prevalence of shedding MDR bacteria was seen in herd $4(52.00 \%, \pm 7.89)$ and the

275 lowest was seen in herd $3(10.00 \% \pm 4.74)$. Herds 1 and 2 each showed the lowest prevalence of

276 shedding AMR bacteria $(37.50 \% \pm 7.65)$, while herd 4 showed the highest prevalence of

277 shedding AMR $(50.00 \%, \pm 7.90)$. The seasonal prevalence of MDR and AMR resistance across

278 three bacterial species is presented in Supplementary Figure 1.

279 The annual prevalence of cows shedding MDR Salmonella was $8.62 \%( \pm 3.65, \mathrm{n}=58)$.

280 The highest prevalence for MDR was detected in the winter season $(10.53 \%, \pm 7.04, \mathrm{n}=19)$ and

281 the highest AMR prevalence was detected in fall $(42.31 \% \pm 9.68, \mathrm{n}=26)$. Salmonella isolated

282 from cows in the summer season did not show any AMR or MDR phenotypes (Figure 1a). The

283 annual prevalence for MDR phenotypes within E. coli isolates was $2.92 \%( \pm 1.09, \mathrm{n}=238)$. The

284 highest prevalence for MDR phenotypes in $E$. coli was detected in winter $(4.08 \% \pm 2.82, \mathrm{n}=49)$

285 and the highest AMR prevalence in E. coli was detected in spring $(34.28 \% \pm 5.67, \mathrm{n}=70$, Figure

286 1b). The annual prevalence for MDR phenotypes within Enterococcus spp. isolates was $26.35 \%$ 
$287( \pm 2.84, \mathrm{n}=238)$. The highest prevalence for MDR Enterococcus spp. was detected in the 288 summer season $(50.00 \% \pm 11.18, \mathrm{n}=20)$ and the highest AMR prevalence in Enterococcus $\mathrm{spp}$. 289 was detected in the winter season $(48.97 \% \pm 7.14, \mathrm{n}=49$, Figure $1 \mathrm{c})$. 
291 Antimicrobial resistance phenotype shared between bacterial isolates:

292 Study found no perfectly shared resistance phenotype between the study isolates. Within the 293 commensal bacteria (E. coli or Enterococcus spp.), resistance to tetracycline was the most 294 prevalent (11 cows), while shared resistance to each of the drugs kanamycin, streptomycin, and 295 chloramphenicol was observed once. However, 6 individual cows were detected sharing 296 tetracycline resistance phenotypes between Salmonella and one or both commensal bacteria $(E$. 297 coli or Enterococcus spp.). Of the 6 cows, five were from a single herd (herd 4) and three shed

298 MDR bacteria (Table 5). However, Salmonella, and one or both commensal species, were found 299 to share resistance to only 1 drug, tetracycline, in 6 cows across the study. Of the 6 cows, only 3 300 had bacterial species with MDR phenotypes detected and in 2 of these cows the MDR profile 301 included tetracycline.

302

303 Model performance and tuning results: Grid search of model parameters with 3-fold cross304 validation yielded satisfactory results in classifying MDR cows based on herd management 305 practices and cow-related features. The sensitivity of models ranged from 0.47 to 0.74 with 306 precision ranging from 0.46 to 0.66 . Details related to hyperparameters, best performing decision 307 tree classifiers, random forest, and XGboost models and the performance of the selected models 308 are given in Table 1.

309

310 Association between herd management practices and cow-related features with shedding 311 multi-drug resistant bacteria

312 Decision tree classification models: 
For all DTC models, the impurity (gini), a measure of the heterogeneity of the outcome in

314 a subset of samples resulting from a split in a decision tree, was reduced in samples the most by

315 the rolling herd average milk production, denoting its highest position in decision trees generated

316 by all five models (Figure1, Figure S2-S5). Other management features that formed nodes

317 showing high measures of split quality (gini) including the proportion of Jersey cows in the herd,

318 administration of tetracyclines, monthly culling percentage, culling frequency, and the number of

319 culled individuals. Of the five DCT models, all but the model for overall resistance across

320 Salmonella and commensal bacterial species were able to generate nodes that classify cows

321 based on their fecal shedding of bacteria to a single class of AMD, or not resistant

322 (Supplementary figures 2-5) with 100\% purity (homogenous subsets). While only the DCT

323 model for commensal bacteria, resulted in pure nodes for MDR cows, none of the other models

324 were able to classify cows into pure MDR nodes (Supplementary figures 2). Figure 2 shows the

325 optimum decision tree for classifying cows into MDR, AMR, or not resistant based on

326 phenotypes of all bacteria (Salmonella, Enterococcus spp., E. coli).

327 Random forest models:

328 Results of random forest models indicated common herd management practices that

329 influence the shedding of MDR and AMR phenotypes of Salmonella, Enterococcus spp., and E.

330 coli collectively, as well as individually (Figure 3). The number of culled animals, monthly

331 culling frequency and percentage, herd size, and proportion of Holstein cows in the herd were

332 found to be influential herd characteristics (top ten features by relative influence) predicting

333 MDR phenotypes in all algorithms. Random forest algorithms for predicting AMR phenotype in

334 Enterococcus spp., commensal bacterial species combined, and in all bacterial species showed

335 the same top ten most influential herd management features. Individual-level features such as 
336 culling due to milk production, mastitis, reproductive and other reasons appeared important for

337 predicting resistance phenotypes in Salmonella. The use of the chalk method for withdrawal

338 determination was in the top ten most influential features and for the E. coli and Salmonella

339 models.

340

\section{Gradient boosting classification models:}

342 Herd characteristics that showed higher variable importance in SHAP values for

343 predicting cows shedding MDR resistance bacteria based on all bacterial species were herd size,

344 the proportion of Jersey cows, sampling season, the frequency of extra-label drug use, rolling

345 herd average, and culling related features. These features also showed high marginal

346 contributions in predicting AMR phenotypes. For predicting AMR phenotypes, features such as

347 the number of monthly veterinary treatments, antibiotic dose tracking, and the proportion of

348 Holstein cows also showed higher SHAP values (Figure 4).

Partial dependence of gradient boosting model prediction on important features showed the

possible relationships of these herd and cow-related features in predicting the AMR of their fecal bacteria as MDR. Higher culling frequency and monthly culling percentages were associated with cows with MDR phenotypes from all bacteria, whereas an overall increase in the number of culled animals from the herd showed a negative trend with classifying a cow as shedding MDR bacteria. Winter season was negatively associated with MDR phenotype bacteria shed by cows compared to cows sampled in Spring. Similarly, herds with more than 10,432 kgs of rolling average milk production showed a positive trend with MDR positive cows. Cows from herds with a higher proportion of Jersey cows were negatively associated with being classified as 
359 shedding MDR bacteria by the gradient boosting algorithm (Figure 5). Within other herd

360 characteristics that were identified as important, herd size showed a varying trend with

361 classifying cows shedding MDR bacteria, with some herd sizes showing a positive association of

362 classifying cows as shedding MDR bacteria (Figure 5).

$363 \quad$ For predicting MDR phenotypes in Salmonella shed by cows, rolling herd average milk

364 production, sampling season, chalk methods for tracking withdrawal, monthly culling frequency, 365 and Salmonella vaccine were found to be important predictive features with high SHAP values

366 (Figure 6). The exploration into partial dependence of these features gave insights into

367 relationships of feature values with classifying a cow as shedding MDR phenotype Salmonella

368 (Figure 7). Increasing rolling herd average milk production and monthly culling percentage was

369 positively associated with MDR phenotypic Salmonella in cow feces. Similarly, cows sampled in 370 spring were more likely to be classified as shedding MDR Salmonella.

371

372

Models predicting phenotypes in commensals E. coli, and Enterococcus spp.; the number

373 of culled animals in the previous year was always in the top ten most important features to

374 classify cows as shedding MDR bacteria (Figure S5- S8). Rolling herd average milk production

375 features describing culling practices, and herd size, consistently featured as important in

376 classifying cows as shedding MDR phenotypic bacteria for all the other three models

377 (Commensals, E. coli, and Enterococcus spp.). The frequency of extra-label drug use was an

378 important feature for models separately predicting MDR in Enterococcus spp. (figure S7) and E.

379 coli shed by cows.

380

381 Discussion 
382 The current study investigated antimicrobial resistance phenotypes between bacteria shed in

383 dairy cattle using decision tree classification algorithms. A simple decision tree model does tend

384 to find the best fit for the training data, but the splitting process rarely is generalizable to other

385 data. A random forest model, which is bagging of decision trees, and a boosting classification

386 model, which is boosting decision trees, tend to perform better on testing data and can help us

387 identify the generalizable conclusion. In this analysis we explored these models step by step

388 from a simple decision tree to generalized boosting trees to find important management-related

389 factors that might affect the distribution of multidrug resistance in dairy cattle herds.

390 A unique aspect of the current study is use of the aforementioned algorithms to distinguish

391 between the resistance profiles (no resistance, antimicrobial resistance and multidrug resistance)

392 of a pathogenic bacteria, Salmonella, and commensal bacteria (E. coli and Enterococcus spp.)

393 isolates from feces of cull dairy cows. Previous investigations were restricted to understanding

394 herd and cow level characteristics with the resistant phenotypes in Salmonella shed by cull dairy

395 cows (Pereira et al. 2019). We explored weather considering the resistance phenotypes for these

396 three bacterial species together, and separately, to identify associations between herd

397 management practices and prevalence of resistance phenotypes. Although, none of the isolates

398 had shared phenotype resistance the six cows that had tetracycline resistance in at least two of

399 the three bacteria studies should be explored further with whole genome sequencing and follow

400 up studies that employ metagenomic analyses on the microbiome.

401 The three machine learning algorithms tested in this study indicated that the overall distribution

402 of three resistance phenotypes classified in this study as MDR, AMR, or no resistance was

403 mainly governed by resistance in Enterococcus spp., which showed the highest prevalence of

404 MDR and AMR phenotypes compared to Salmonella and E. coli. The latter may be explained by 
405 Enterococcus spp. that are known to have frequent MDR phenotypes such as E. faecium.

406 Comparative feature importance plots for all random forest models developed for these bacterial

407 groups indicated the same, where similar features are found to be important for the model

408 predicting MDR in all bacteria together, in commensals together, and in Enterococcus spp..

409 Herd size has been already identified as associated with higher odds of detecting Salmonella

410 resistant to tetracycline (Pereira et al. 2019). In the current study, we showed that herd size was

411 also positively associated with detecting MDR phenotypes in Salmonella as well as the

412 commensal bacteria E. coli and Enterococcus spp. Salmonellosis is known to be associated with

413 poor milk production and reproduction and hence increased risk for diseases, such as mastitis

414 and infertility, and AMD treatments which may explain the 12\% MDR in Salmonella isolates

415 from the study cows (Lanzas et al. 2010). Similarly, Salmonella has been associated with clinical

416 disease in both adult and young dairy cattle and beef cows (Divers \& Peek 2007; Pender 2003;

417 Roy et al. 2001; Smith 2014). Percent cull and rate would reflect the increase in culling due to

418 diseases better than actual numbers culled. This is evident from the importance of all three in the

419 random forest model for Salmonella MDR phenotypes. In contrast, the total number culled was

420 negatively associated with shedding multidrug resistant bacteria which may reflect producer

421 decisions to prioritize the culling of otherwise healthy but low-producing cows based on milk or

422 beef market value (with respect to dairy beef), amongst other factors. Other than market

423 dynamics including milk or beef demand, underlying herd health or management reasons may

424 explain the opposing trends of both percent and number culled, and the outcome MDR in cull

425 cow fecal bacteria. Herds with MDR in their cull cow fecal bacteria hence need to be explored

426 further to identify mechanisms that eventually increase culling rates. It is worthy to note that the

427 random forest algorithm identified diseases of relative importance for MDR in Salmonella but 
428 not commensals. However, caution should be exercised with interpreting findings from this 429 specific analysis due to the inability to ascertain that such diseases preceded the Salmonella 430 shedding and specifically MDR status. The combined analysis of all 3 species MDR random 431 forest however did not show diseases as correlated with MDR, which may be due to the overall 432 effect of the commensals in the dataset.

433 SHAP values ranked variables by importance for classifying resistance type (either MDR, AMR, 434 or no resistance). However, in the case of rolling herd average milk production (RHA), SHAP 435 values were allocated only for predicting MDR or AMR in models for Salmonella and 436 Enterococcus and commensals, showing zero importance in predicting absence of any resistance.

437 The RHA is hence more important in terms of identifying any resistance type (MDR or AMR) 438 versus no resistance. All three algorithms (DCT, RF and XGboost) indicated a high importance 439 of RHA in predicting MDR in fecal bacteria of cull cows. RHA is a dairy performance indicator 440 affected by multiple herd characteristics such as age and breed structure. Studies have indicated 441 stress related to higher production which may increase the chance of certain health conditions

442 subsequently increasing the risk of antimicrobial drug use and hence bacterial resistance 443 (Robbins et al. 2016). In addition, the association between RHA and MDR presented by all the 444 models here is conditioned upon other features that follow the splits further down the 445 classification tree. In contrast, for E. coli, RHA was important in identifying all resistance 446 classifications (AMR, MDR, no resistance).

447 XGBoost results show that season was correlated with MDR in all 3 species. Specifically, that 448 Spring and Fall had a greater correlation with MDR compared to Summer and Winter, with 449 Winter being the least correlated. Similar findings have been observed with a risk of disease in 450 calves in Spring and Fall with bovine respiratory disease (Cummings et al. 2019; Dubrovsky et 
451 al. 2019; Maier et al. 2019). The current study's bacterial species, specifically Salmonella, E. coli 452 and Enterococcus spp., shared no specific MDR profiles; however, shared tetracycline resistance 453 was detected. Further studies employing whole genome sequencing and metagenomics on the 454 microbiome may explore factors that explain such shared resistance.

455

456

\section{Conclusions}

458 The current study characterized dairy cattle herd management practices that were associated with 459 fecal shedding of multi-drug resistant bacteria. We identified generalizable and distant 460 associations between pathogenic Salmonella and commensal bacteria. The data-driven suite of 461 machine learning algorithms used here can help develop data-informed tools for better decision 462 making, and risk assessment related to antibacterial resistant shedding by cows. 


\section{Acknowledgments}

464 The authors acknowledge the study dairies' owners, herd managers, and staff from

465 California dairies participating in the study. Any opinions, findings, conclusions, or

466 recommendations expressed in this publication are those of the author(s) and do not necessarily

467 reflect the view of the U.S. Department of Agriculture.

\section{Funding}

469 Funding for this research was provided by the U.S. Department of Agriculture Project number

470 CA-V-PHR-4707-AH407 and CA-V-PHR-4048-H. This study was also partially

471 supported by the USDA National Institute of Food and Agriculture (NIFA) project

472 number 201567030238928. The funders had no role in study design, data collection, and

473 analysis, decision to publish, or preparation of the manuscript.

\section{Grant Disclosures}

475 The following grant information was disclosed by the authors:

476 USDA Project number: CA-V-PHR-4707-AH407 and CA-V-PHR-4048-H.

477 USDA National Institute of Food and Agriculture (NIFA) project number: 201567030238928.

\section{Competing interests}

479 The authors declare that they have no competing interests.

\section{Animal Ethics}

481 The study was approved by the University of California, Davis's Institutional Animal Care and

482 Use Committee (protocol number 18019).

\section{Data Availability}

484 The de-identified data was shared for peer review only as the dairy owners did not

485 consent to publish it alongside the article. 


\section{References}

487

488

489

490

491

492

493

494

495

496

497

498

499

500

501

502

503

504

505

506

507

508

509

510

511

512

513

514

515

516

517

518

519

520

521

522

523

524

525

526

527

528

529

530

531

532

Abu Aboud OA, Adaska JM, Williams DR, Rossitto PV, Champagne JD, Lehenbauer TW, Atwill $\mathrm{R}, \mathrm{Li}$ X, and Aly SS. 2016. Epidemiology of Salmonella sp. in California cull dairy cattle: prevalence of fecal shedding and diagnostic accuracy of pooled enriched broth culture of fecal samples. PeerJ 4:e2386. 10.7717/peerj.2386

Adaska JM, Ekong PS, Clothier KA, Williams DR, Rossitto PV, Lehenbauer TW, Atwill ER, Li X, and Aly SS. 2020. Bayesian estimation of diagnostic accuracy of fecal culture and PCRbased tests for the detection of Salmonella enterica in California cull dairy cattle. PeerJ 8:e8310. 10.7717/peerj.8310

Aidara-Kane A, Angulo FJ, Conly JM, Minato Y, Silbergeld EK, McEwen SA, and Collignon PJ. 2018. World Health Organization (WHO) guidelines on use of medically important antimicrobials in food-producing animals. Antimicrobial Resistance \& Infection Control $7: 7$.

Breiman L. 2001. Random forests. Machine learning 45:5-32.

Chen T, and Guestrin C. 2016. Xgboost: A scalable tree boosting system. Proceedings of the 22nd acm sigkdd international conference on knowledge discovery and data mining: ACM. p 785-794.

CLSI. 2014. Performance standards for antimicrobial disk and dilution susceptibility tests for bacteria isolated from animals: approved standard: NCCLC.

CLSI. 2018. M100 Performance standards for antimicrobial susceptibility testing: Clinical and Laboratory Standards Institute Wayne, PA.

Control CfD, and Prevention. 2019. Antibiotic resistance threats in the United States, 2019: Centres for Disease Control and Prevention, US Department of Health and Services, CDC.

Cummings KJ, Rodriguez-Rivera LD, Capel MB, Rankin SC, and Nydam DV. 2019. Oral and intranasal administration of a modified-live Salmonella Dublin vaccine in dairy calves: Clinical efficacy and serologic response. Journal of dairy science 102:3474-3479.

Divers TJ, and Peek SF. 2007. Rebhun's diseases of dairy cattle: Elsevier Health Sciences.

Dubrovsky S, Van Eenennaam A, Karle B, Rossitto P, Lehenbauer TW, and Aly SS. 2019. Epidemiology of bovine respiratory disease (BRD) in preweaned calves on California dairies: The BRD 10K study. Journal of dairy science 102:7306-7319.

Elith J, Leathwick JR, and Hastie T. 2008. A working guide to boosted regression trees. J Anim Ecol 77:802-813. 10.1111/j.1365-2656.2008.01390.x

Friedman JH. 2001. Greedy function approximation: A gradient boosting machine. Annals of statistics 29:1189-1232. DOI 10.1214/aos/1013203451

Grimont PA, and Weill F-X. 2007. Antigenic formulae of the Salmonella serovars. WHO collaborating centre for reference and research on Salmonella 9:1-166.

Hyde RM, Down PM, Bradley AJ, Breen JE, Hudson C, Leach KA, and Green MJ. 2020. Automated prediction of mastitis infection patterns in dairy herds using machine learning. Scientific reports 10:1-8.

Lanzas C, Warnick LD, James KL, Wright EM, Wiedmann M, and Grohn YT. 2010. Transmission dynamics of a multidrug-resistant Salmonella typhimurium outbreak in a dairy farm. Foodborne Pathog Dis 7:467-474. 10.1089/fpd.2009.0411

Li X, Aly S, Su Z, Pereira R, Williams D, Rossitto P, Champagne J, Chase J, Nguyen T, and Atwill E. 2018. Phenotypic antimicrobial resistance profiles of E. coli and Enterococcus from dairy cattle in different management units on a central California dairy. Clin Microbiol 7:1-7. 
533

534

535

536

537

538

539

540

541

542

543

544

545

546

547

548

549

550

551

552

553

554

555

556

557

558

559

560

561

562

563

564

565

566

567

568

569

570

Li X, Watanabe N, Xiao C, Harter T, McCowan B, Liu Y, and Atwill ER. 2014. Antibiotic-resistant E. coli in surface water and groundwater in dairy operations in Northern California. Environmental monitoring and assessment 186:1253-1260.

Lundberg SM, and Lee S-I. 2017. A unified approach to interpreting model predictions. Advances in Neural Information Processing Systems. p 4765-4774.

Machado G, Mendoza MR, and Corbellini LG. 2015. What variables are important in predicting bovine viral diarrhea virus? A random forest approach. Veterinary research 46:85.

Maier G, Love W, Karle B, Dubrovsky S, Williams D, Champagne J, Anderson R, Rowe JD, Lehenbauer TW, and Van Eenennaam A. 2019. Management factors associated with bovine respiratory disease in preweaned calves on California dairies: The BRD 100 study. Journal of dairy science 102:7288-7305.

Mollenhorst H, Ducro BJ, De Greef KH, Hulsegge I, and Kamphuis C. 2019. Boosted trees to predict pneumonia, growth, and meat percentage of growing-finishing pigs. Journal of animal science 97:4152-4159.

Pedregosa F, Varoquaux G, Gramfort A, Michel V, Thirion B, Grisel O, Blondel M, Prettenhofer P, Weiss R, Dubourg V, Vanderplas J, Passos A, Cournapeau D, Brucher M, Perrot M, and Duchesnay E. 2011. Scikit-learn: Machine Learning in Python. Journal of machine learning research 12:2825-2830.

Pender AB. 2003. Salmonellosis in a herd of beef cows. The Canadian Veterinary Journal 44:319.

Pereira R, Williams DR, Rossitto P, Adaska J, Okello E, Champagne J, Lehenbauer TW, Li X, Chase J, Nguyen T, Pires AFA, Atwill ER, and Aly SS. 2019. Association between herd management practices and antimicrobial resistance in Salmonella spp. from cull dairy cattle in Central California. PeerJ 7:e6546. 10.7717/peerj.6546

Quinn P, Markey BK, Carter M, Donnelly W, and Leonard F. 2002. Veterinary microbiology and microbial disease: Blackwell science.

Robbins J, Von Keyserlingk M, Fraser D, and Weary D. 2016. Invited review: Farm size and animal welfare. Journal of animal science 94:5439-5455.

Roy R, Higgins R, Fortin M, and Tardif S. 2001. Salmonella Give infection in 2 dairy herds. The Canadian Veterinary Journal 42:468.

Scallan E, Hoekstra RM, Angulo FJ, Tauxe RV, Widdowson M-A, Roy SL, Jones JL, and Griffin PM. 2011. Foodborne illness acquired in the United States-major pathogens. Emerging infectious diseases 17:7.

Smith BP. 2014. Large animal internal medicine-E-Book: Elsevier Health Sciences.

Troutt HF, Galland JC, Osburn BI, Brewer RL, Braun RK, Schmitz JA, Sears P, Childers AB, Richey E, and Mather E. 2001. Prevalence of Salmonella spp in cull (market) dairy cows at slaughter. Journal of the American Veterinary Medical Association 219:1212-1215.

Peer] reviewing PDF | (2021:01:57027:2:0:NEW 24 May 2021) 


\section{Figure 1}

Seasonal variation in the prevalence of multidrug antimicrobial resistance

Seasonal variation in the prevalence of multidrug antimicrobial resistance (MDR; resistance to 3 or more drug classes), and antimicrobial resistance (AMR; resistance to 1 or 2 drug classes only) in Salmonella (a), E. coli (b), and Enterococcus spp. (c) isolates from six California dairy herds. Orange and green dashed lines show the annual average prevalence of MDR and AMR in all six herds respectively. The proportion of cows that did not show any resistance are the inverse of the sum of MDR and AMR proportions and not shown in the figure. Point estimates and single standard error deviation are represented by circles and whiskers respectively. 

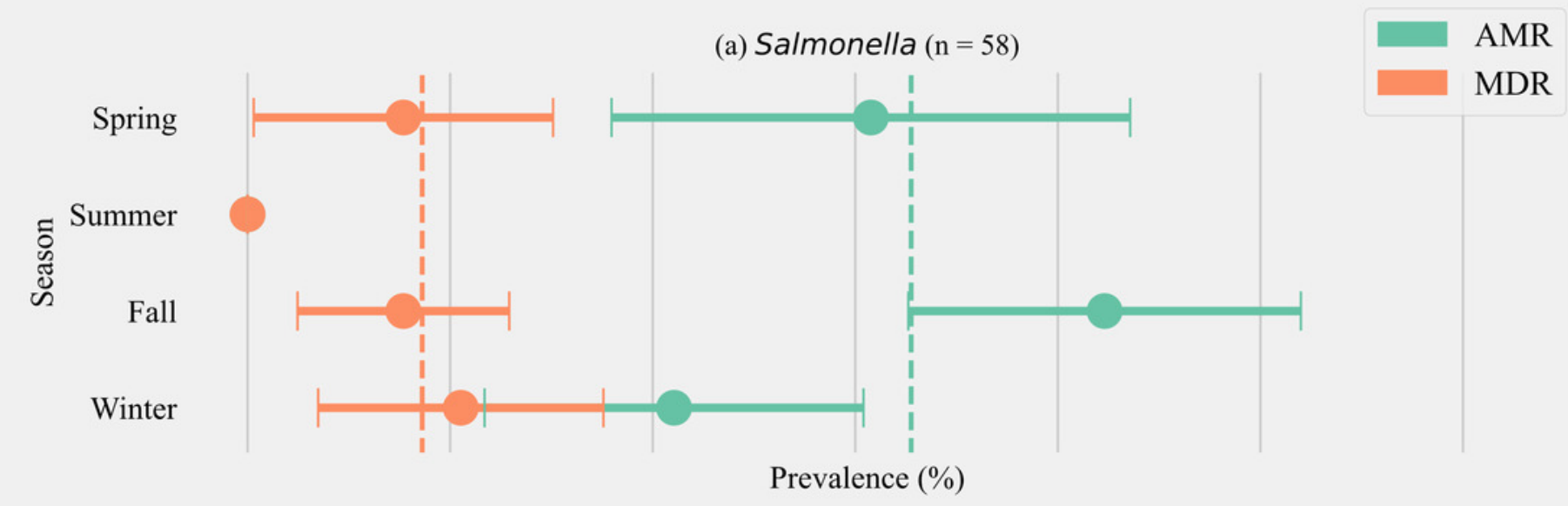

(b) E. coli $(\mathrm{n}=238)$

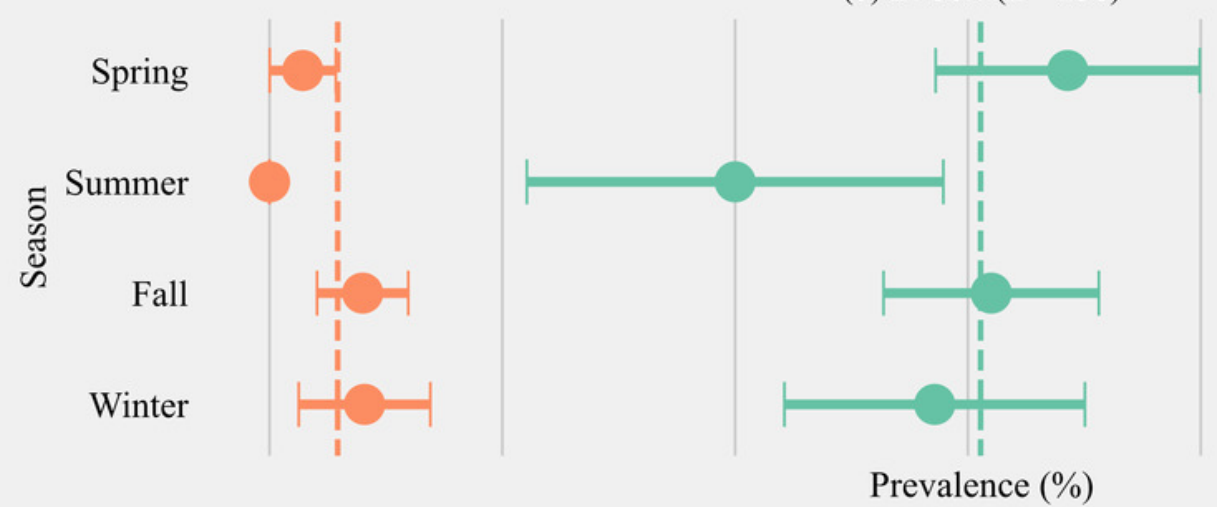

(c) Enterococcus spp. $(\mathrm{n}=238)$

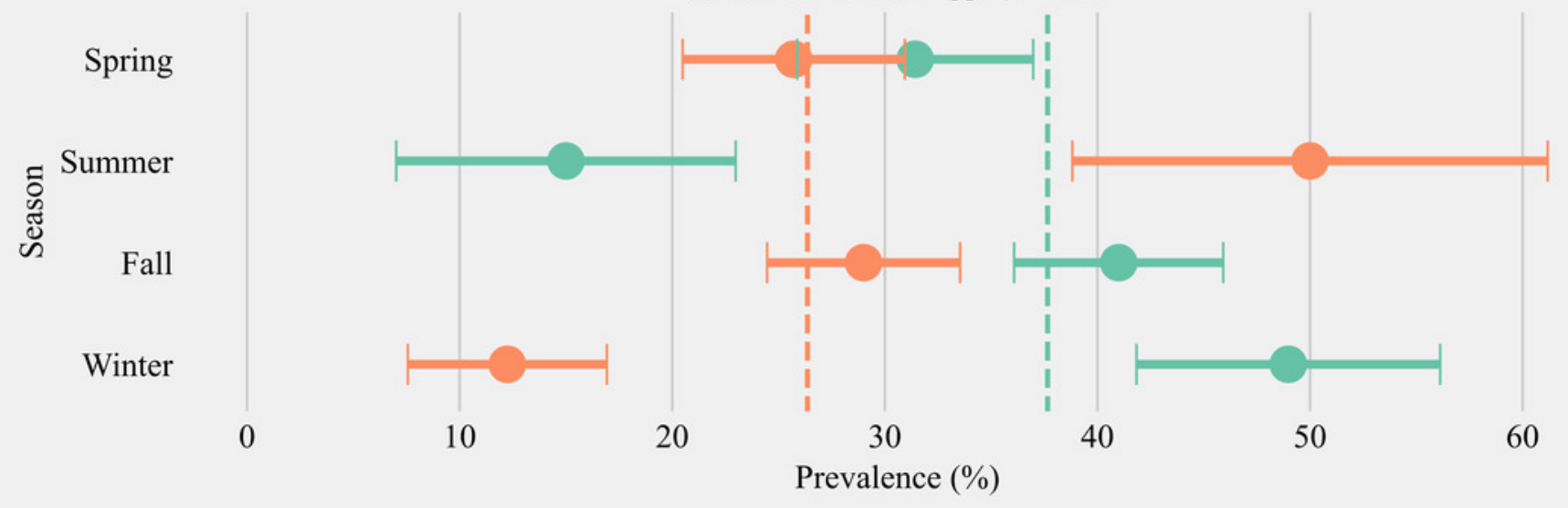




\section{Figure 2}

Optimum decision tree to classify cows shedding multi-drug resistant (MDR), antimicrobial-resistant (AMR), and non-resistant Salmonella, Enterococcus spp., E. coli based on management practices observed in Californian dairy herds.

Nodes are represented by stacked histograms depicting distribution samples in the data (AMR, MDR, no resistance), followed by optimum decision point (pointer on the x-axis). Arrows on the left and right indicate lesser and greater than the decision point respectively. Final nodes are represented by pie chart with distribution of samples. Factor acronym definitions are described in Table 2. 


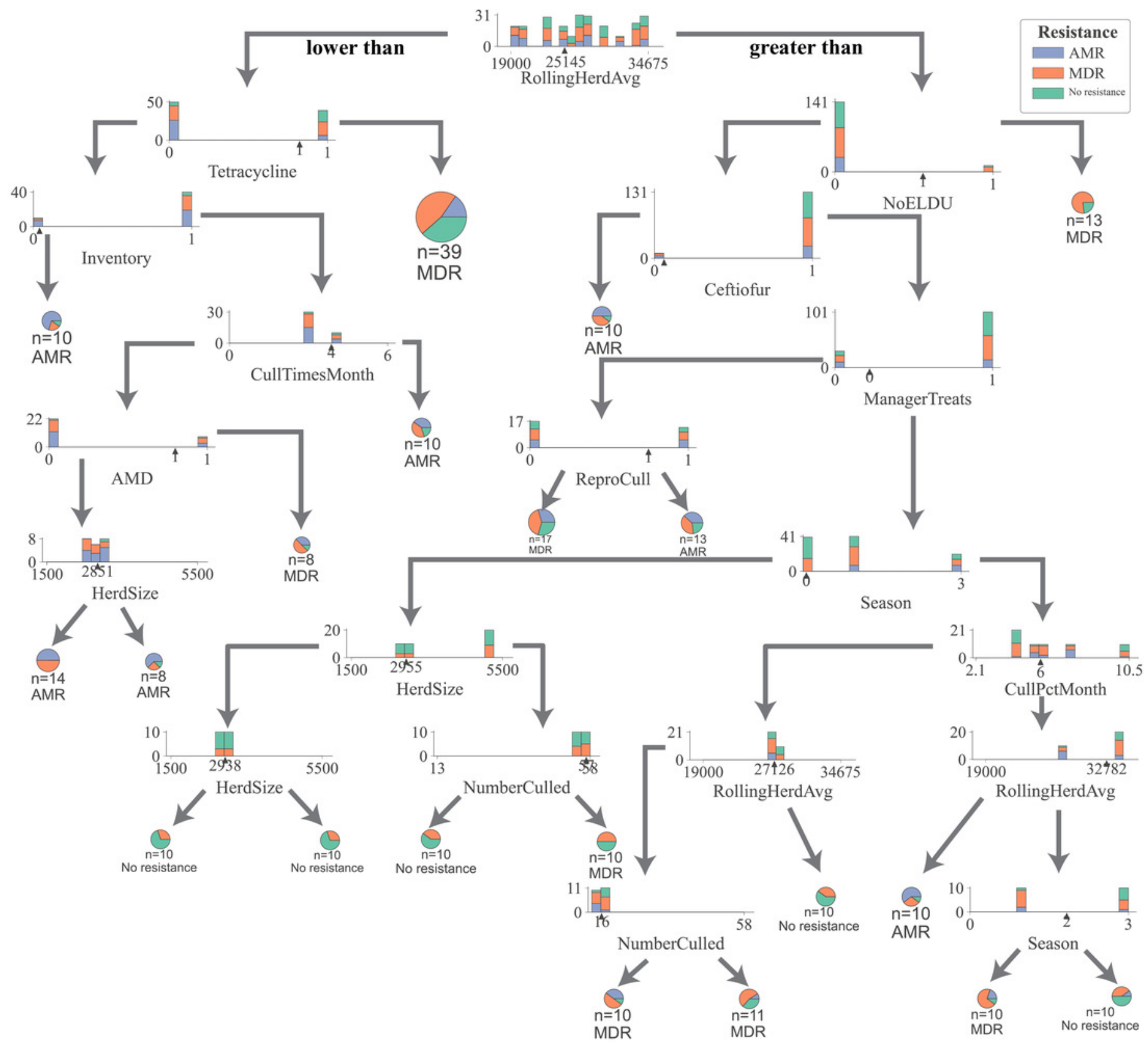


Figure 3

Top ten herd management practices based on variable importance (Gini coefficient) in classifying cows shedding multi-drug resistant (MDR), antimicrobial-resistant (AMR), and non-resistant for Salmonella, Enterococcus spp., E. coli in Cal

Factor acronym definitions described in Table 2.

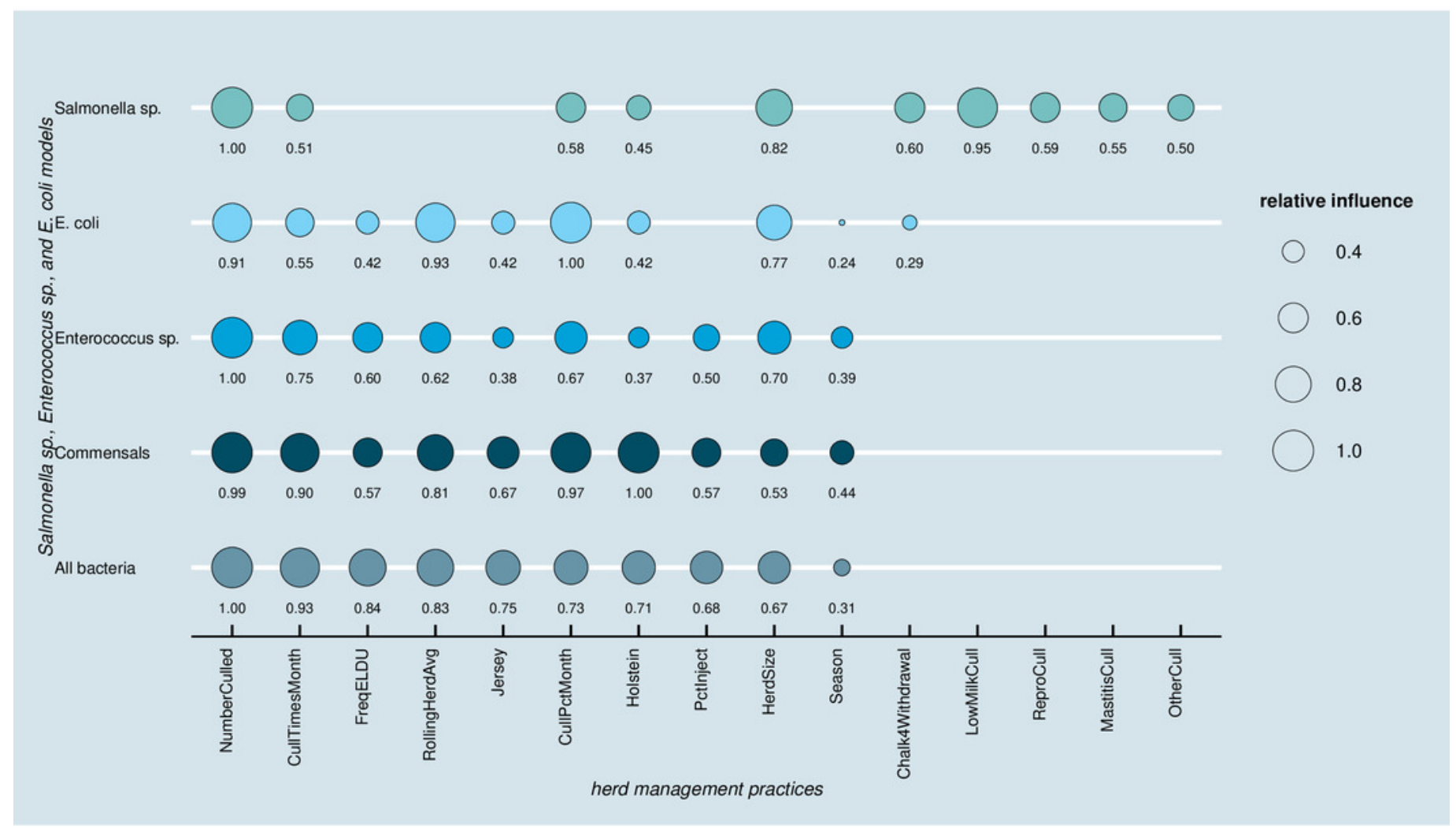


Figure 4

Mean SHAP values depicting the impact of herd management practices on predicting multi-drug resistant phenotype in either Salmonella, Enterococcus spp., and E. coli shed in dairy cows for Gradient boosting classification (XGboost) model.

Factor acronym definitions described in Table 2. 


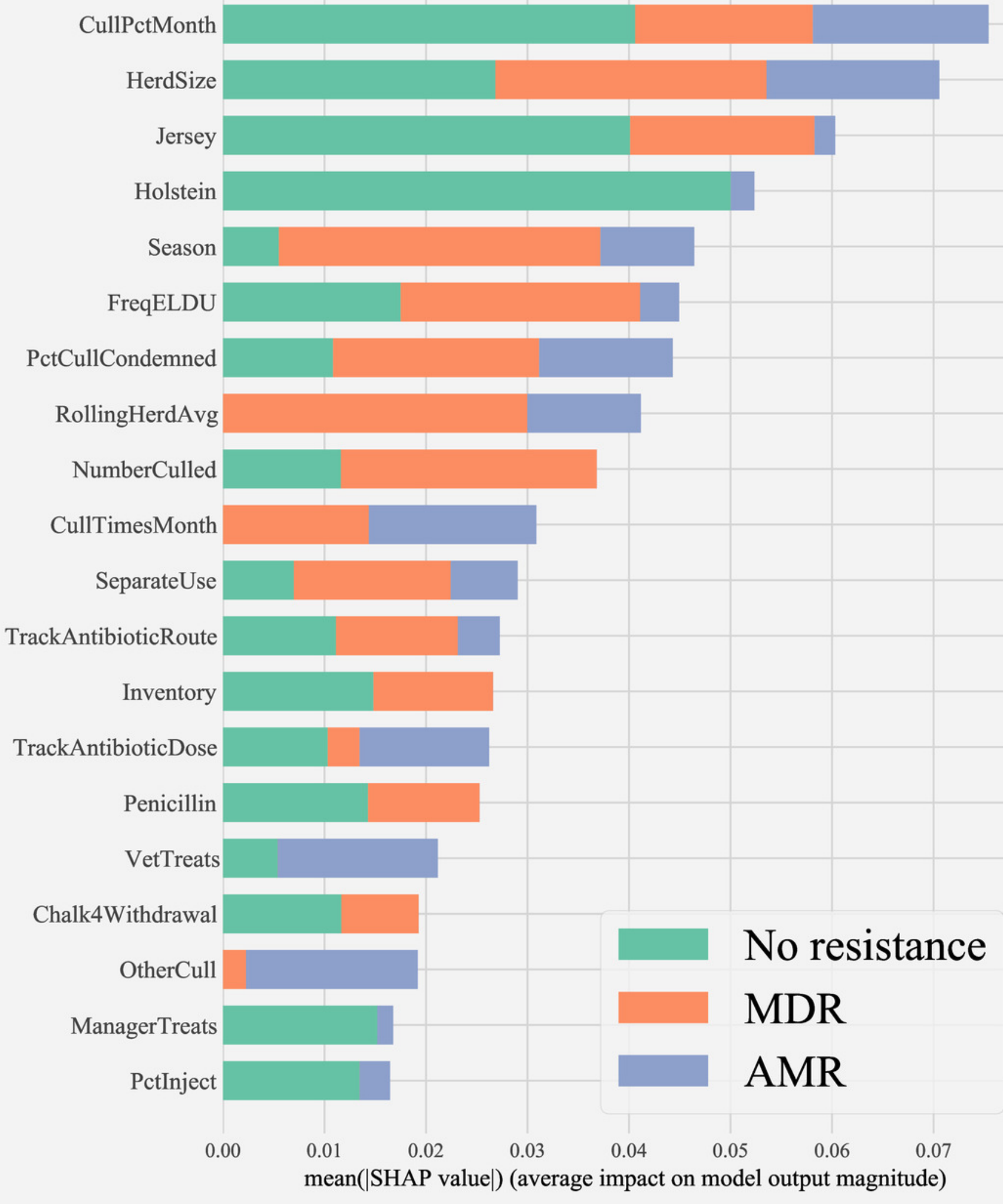




\section{Figure 5}

Partial dependence indicating the association of top six predictive herd management practices in classifying cows as multi-drug resistant phenotype in either Salmonella, Enterococcus spp. and E. coli shed in dairy cows for Gradient boost

Partial dependence plots are generated for values presented in the data resulting in the nonlinear $\mathrm{x}$-axis. Blue shaded region and error bars represents standard deviation of partial dependence $(n=238)$.

(a)

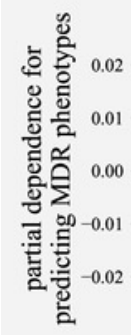

$\begin{array}{llllllllll}2.1 & 2.83 & 3.17 & 3.25 & 4.5 & 4.6 & 5.3 & 7.1 & 8.6 & 10.5\end{array}$ Monthly culling percentage

(d)

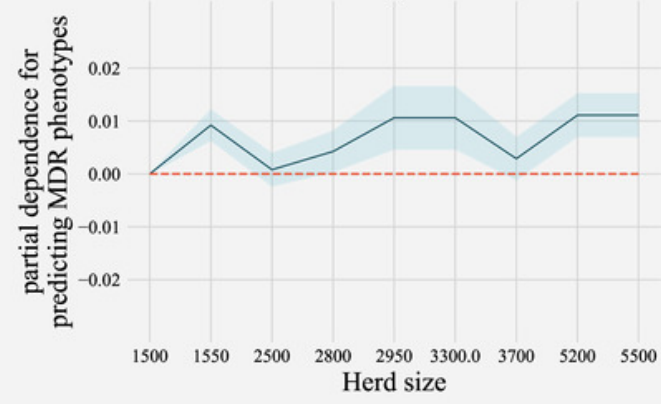

(b)

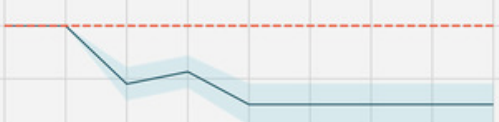

13

$\begin{array}{lll}18 & 21 & 29 \\ \text { Number of culled animals }\end{array}$

(e)

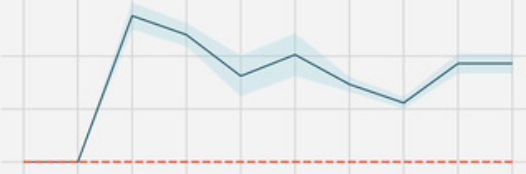

(c)
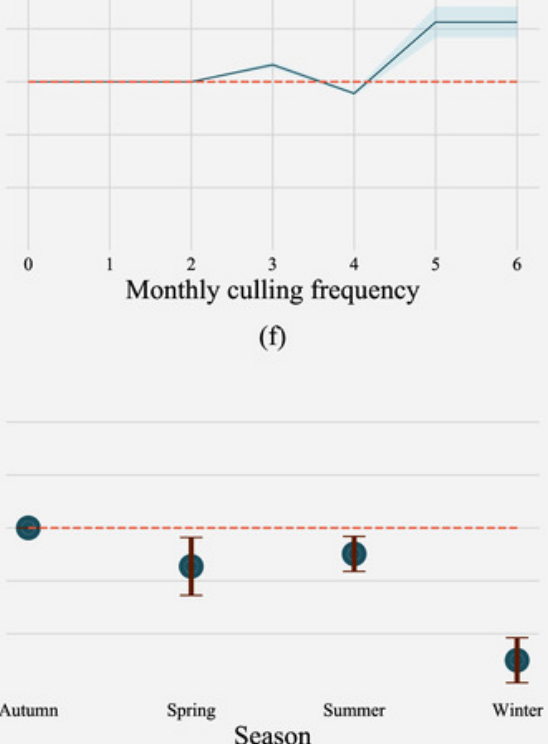

19000208002350024800266002737529200310253390034675 Autumn Rolling herd average milk productions

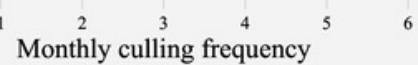

(f) Season 
Figure 6

Mean SHAP values depicting the impact of herd management practices on predicting multi-drug resistant phenotype in Salmonella shed in dairy cows for Gradient boosting classification (XGboost) model.

Factor acronym definitions described in Table 2. 
HerdSize

RollingHerdAvg

Holstein

Season

Chalk4Withdrawal

CullPctMonth

CombinationUse

PctInject

Jersey

AMD

SalmonellaVaccine

Anti-Inf
No-Treatment

Other

Tetracycline

Penicillin

Ceftiofur

OtherCull

MastitisCull

LameCull

MDR

AMR

No resistance

0.00

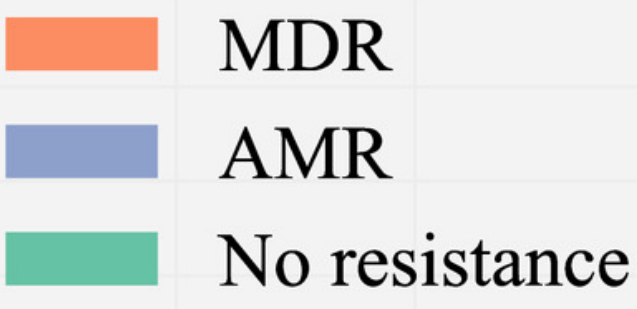

$0.04 \quad 0.06$

0.08

0.10

mean(|SHAP value|) (average impact on model output magnitude) 


\section{Figure 7}

Partial dependence indicating the association of top-six predictive herd management practices in classifying cows as multi-drug resistant phenotype in Salmonella shed in dairy cows for Gradient boosting classification (XGboost) model.

Partial dependence plots are generated for values presented in the data resulting in the nonlinear $\mathrm{x}$-axis. Blue shaded region and error bars represent the standard deviation of partial dependence $(n=58)$.

(a)

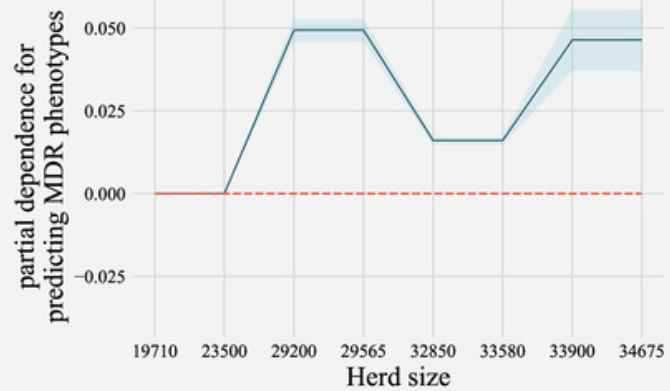

(d)

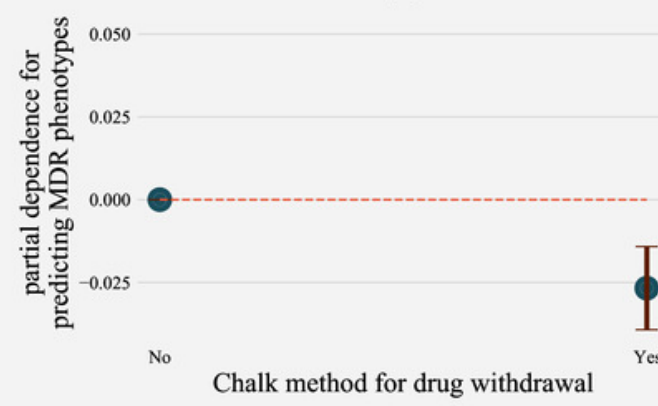

$\oint$ (b)

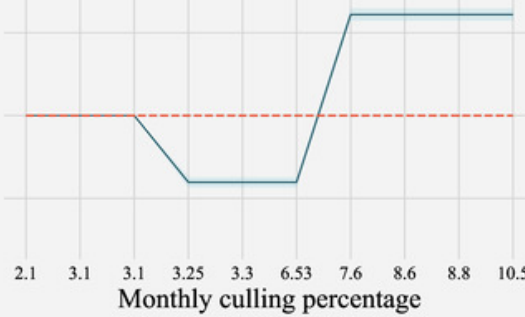

(e) (c)

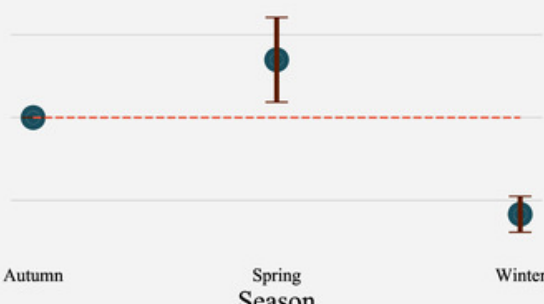

(f)

$\Phi$

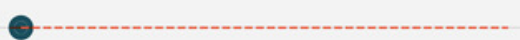

No

Salmonella vaccine

Automated mastitis detection 


\section{Table $\mathbf{1}$ (on next page)}

Hypertuning of model parameters and validation

Classification algorithms trained and tested to predict multidrug resistance phenotypes from bacterial isolates for bacterial species and groups of bacteria. Parenthesis ( $n$ ) sample size of the number of cows for each model. For each bacterial and bacterial group model, hypertuning of the decision tree classifier, random forest, and XGBoost models is presented. The table shows parameters tuned, values tested for tuning models, best model parameters, and the performance of the selected model in terms of precision, recall and f1-score for the holdout dataset. 


\begin{tabular}{|c|c|c|c|c|c|c|c|}
\hline \multirow[t]{2}{*}{ Model parameters } & \multicolumn{2}{|c|}{$\begin{array}{l}\text { Output definition based on resistance for number } \\
\text { of antimicrobial classes }\end{array}$} & \multirow[t]{2}{*}{$\begin{array}{l}\text { Salmonella } \\
(\mathrm{n}=\mathbf{5 8})\end{array}$} & \multirow[t]{2}{*}{$\begin{array}{l}\text { E. coli } \\
(\mathbf{n}=\mathbf{2 3 8})\end{array}$} & \multirow[t]{2}{*}{$\begin{array}{l}\text { Enterococcus spp. } \\
(\mathbf{n}=\mathbf{2 3 8})\end{array}$} & \multirow[t]{2}{*}{$\begin{array}{l}\text { Combined resistance } \\
\text { in } \text { E. coli and } \\
\text { Enterococcus } \text { spp. } \\
(\mathbf{n}=\mathbf{2 3 8})\end{array}$} & \multirow[t]{2}{*}{$\begin{array}{l}\text { Resistance in } \\
\text { antimicrobial classes in } \\
\text { either Salmonella or } E \text {. } \\
\text { coli } \text { or Enterococcus spp. } \\
(\mathbf{n}=\mathbf{2 3 8})\end{array}$} \\
\hline & Parameter explanation & $\begin{array}{l}\text { Parameter } \\
\text { values tested }\end{array}$ & & & & & \\
\hline \multicolumn{8}{|c|}{ Decision tree classifier } \\
\hline Criterion & $\begin{array}{l}\text { The function to measure the } \\
\text { quality of a split }\end{array}$ & gini, entropy & gini & entropy & entropy & gini & gini \\
\hline Splitter & $\begin{array}{l}\text { The strategy used to choose the } \\
\text { split at each node. }\end{array}$ & best, random & best & best & best & random & random \\
\hline Maximum depth & The maximum depth of the tree & $\begin{array}{l}10,20,30,40 \\
45,50,70\end{array}$ & 10 & 45 & 50 & 45 & 10 \\
\hline Minimum split & $\begin{array}{l}\text { The minimum number of samples } \\
\text { required to split an internal node }\end{array}$ & $2,3,4,6$ & 6 & 6 & 6 & 3 & 3 \\
\hline Maximum features & $\begin{array}{l}\text { The number of features to } \\
\text { consider when looking for the } \\
\text { best split }\end{array}$ & auto, sqrt & auto & auto & auto & sqrt & sqrt \\
\hline Minimum leaf & $\begin{array}{l}\text { The minimum number of samples } \\
\text { required to be at a leaf node }\end{array}$ & $1,3,4,6,7,8$ & 4 & 4 & 6 & 1 & 7 \\
\hline \multicolumn{8}{|c|}{ Model performance for holdout dataset } \\
\hline $\mathrm{n}$ & $\begin{array}{l}\text { number of samples in holdout } \\
\text { dataset }\end{array}$ & & 19 & 73 & 73 & 73 & 73 \\
\hline Precision & positive predictive value & & 0.72 & 0.65 & 0.51 & 0.44 & 0.47 \\
\hline Recall & sensitivity & & 0.79 & 0.68 & 0.52 & 0.44 & 0.47 \\
\hline F1-score & $\begin{array}{l}\text { harmonic mean of PPV and } \\
\text { sensitivity }\end{array}$ & & 0.75 & 0.66 & 0.50 & 0.44 & 0.47 \\
\hline \multicolumn{8}{|l|}{ Random forest } \\
\hline Bootstrap & $\begin{array}{l}\text { Whether bootstrap samples are } \\
\text { used when building trees }\end{array}$ & True, False & FALSE & TRUE & TRUE & TRUE & TRUE \\
\hline Criterion & $\begin{array}{l}\text { The function to measure the } \\
\text { quality of a split }\end{array}$ & gini, entropy & gini & entropy & entropy & entropy & entropy \\
\hline Maximum depth & The maximum depth of the tree & $\begin{array}{l}10,20,30,40 \\
45,50,70\end{array}$ & 30 & 50 & 30 & 40 & 10 \\
\hline
\end{tabular}




\begin{tabular}{|c|c|c|c|c|c|c|c|}
\hline Maximum features & $\begin{array}{l}\text { The number of features to } \\
\text { consider when looking for the } \\
\text { best split }\end{array}$ & auto, sqrt & sqrt & sqrt & sqrt & auto & auto \\
\hline Minimum leaf & $\begin{array}{l}\text { The minimum number of samples } \\
\text { required to be at a leaf node }\end{array}$ & $1,3,4,6,7,8$ & 1 & 8 & 4 & 8 & 8 \\
\hline Minimum split & $\begin{array}{l}\text { The minimum number of samples } \\
\text { required to split an internal node }\end{array}$ & $2,3,4,6$ & 3 & 3 & 2 & 4 & 4 \\
\hline $\begin{array}{l}\text { Number of } \\
\text { estimators }\end{array}$ & The number of trees in the forest & $\begin{array}{l}100,200,300 \\
500\end{array}$ & 200 & 100 & 100 & 100 & 100 \\
\hline \multicolumn{8}{|c|}{ Model performance for holdout dataset } \\
\hline $\mathrm{n}$ & $\begin{array}{l}\text { number of samples in holdout } \\
\text { dataset }\end{array}$ & & 19 & 73 & 73 & 73 & 73 \\
\hline Precision & positive predictive value & & 0.66 & 0.62 & 0.46 & 0.47 & 0.41 \\
\hline Recall & sensitivity & & 0.74 & 0.67 & 0.47 & 0.48 & 0.41 \\
\hline F1-score & $\begin{array}{l}\text { harmonic mean of PPV and } \\
\text { sensitivity }\end{array}$ & & 0.69 & 0.61 & 0.46 & 0.47 & 0.38 \\
\hline \multicolumn{8}{|c|}{ Gradient boosting (XGBoost framework) } \\
\hline Column sample & $\begin{array}{l}\text { Subsample ratio of columns when } \\
\text { constructing each tree }\end{array}$ & $\begin{array}{l}0.2,0.1,0.15,0.4 \\
0.7\end{array}$ & 0.1 & 0.2 & 0.2 & 0.4 & 0.1 \\
\hline Gamma & $\begin{array}{l}\text { Minimum loss reduction required } \\
\text { to make a further partition on a } \\
\text { leaf node of the tree }\end{array}$ & $\begin{array}{l}0.0,0.1,0.2,0.4 \\
0.45,0.5,0.6,0.7\end{array}$ & 0.1 & 0.1 & 0.2 & 0.6 & 0.5 \\
\hline Learning rate & Boosting learning rate & $\begin{array}{l}0.001,0.002 \\
0.005,0.008 \\
0.01,0.02,0.05 \\
0.10,0.25,0.5\end{array}$ & 0.25 & 0.5 & 0.008 & 0.5 & 0.05 \\
\hline Maximum depth & $\begin{array}{l}\text { Maximum tree depth for base } \\
\text { learners }\end{array}$ & $\begin{array}{l}3,4,7,8,9,10 \\
15,20\end{array}$ & 3 & 8 & 8 & 7 & 7 \\
\hline $\begin{array}{l}\text { Minimum child } \\
\text { weight }\end{array}$ & $\begin{array}{l}\text { Minimum sum of instance weight } \\
\text { (hessian) needed in a child }\end{array}$ & $1,3,5,7$ & 3 & 1 & 1 & 1 & 7 \\
\hline $\begin{array}{l}\text { Number of } \\
\text { estimators }\end{array}$ & Number of boosting rounds & $\begin{array}{l}3,5,10,30,40 \\
50,100\end{array}$ & 5 & 30 & 10 & 3 & 30 \\
\hline Objective & $\begin{array}{l}\text { Learning task, binary, multiple, } \\
\text { etc. }\end{array}$ & multi:softprob & $\begin{array}{l}\text { multi:soft } \\
\text { prob }\end{array}$ & multi:softprob & multi:softprob & multi:softprob & multi:softprob \\
\hline \multicolumn{8}{|c|}{ Model performance for holdout dataset } \\
\hline $\mathrm{n}$ & $\begin{array}{l}\text { number of samples in holdout } \\
\text { dataset }\end{array}$ & & 19 & 73 & 73 & 73 & 73 \\
\hline Precision & positive predictive value & & 0.61 & 0.59 & 0.51 & 0.49 & 0.46 \\
\hline Recall & sensitivity & & 0.68 & 0.6 & 0.51 & 0.48 & 0.44 \\
\hline F1-score & harmonic mean of PPV and & & 0.65 & 0.59 & 0.51 & 0.48 & 0.38 \\
\hline
\end{tabular}




\section{PeerJ}

sensitivity 
Table 2 (on next page)

Predictive features

Dairy cattle and herd related features used as predictors in classification models. 


\begin{tabular}{|c|c|}
\hline Cow related features & Herd related features \\
\hline Low milk production cull (LowMilkCull) & Milking herd size (HerdSize) \\
\hline Reproduction cull (ReproCull) & Milk production level (RollingHerdAvg) \\
\hline Lameness cull (LameCull) & Holstein Breed (Holstein) \\
\hline Mastitis cull (MastitisCull) & Jersey Breed (Jersey) \\
\hline Other reasons cull (OtherCull) & Percent culled monthly (CullPctMonth) \\
\hline Antimicrobial Drug Use for cull condition (AMD) & Times culled monthly (CullTimesMonth) \\
\hline Anti-inflammatory treatment for condition (Ani-Inf) & Main cull reason disease \\
\hline No-Treatment for condition (No-Treatment) & Percent culled sold for beef (PctCullBeef) \\
\hline \multirow[t]{21}{*}{ Other treatment for condition (Other) } & Percent carcasses condemned (PctCullCondemned) \\
\hline & Percent culled injected within $2 \sim 3$ weeks (PctInject) \\
\hline & Veterinarian gives sick cow treatments (VetTreats) \\
\hline & Dairy manager gives sick cow treatments (ManagerTreats) \\
\hline & Staff gives sick cow treatments (StaffTreats) \\
\hline & Prevent Residue by avoiding specific drugs (ResiduePrevent) \\
\hline & Chalk on cows to track drug withdrawal (Chalk4Withdrawal) \\
\hline & Keep drug inventory (Inventory) \\
\hline & Penicillin \\
\hline & Ceftiofur \\
\hline & Tetracycline \\
\hline & Antibiotics used separately (SeparateUse) \\
\hline & Antibiotics combinations used (CombinationUse) \\
\hline & Track antibiotic dose used (TrackAntibioticDose) \\
\hline & Track antibiotic route used (TrackAntibioticRoute) \\
\hline & Familiarity with ELDU (FamiliarELDU) \\
\hline & Frequency of ELDU (FreqELDU) \\
\hline & No ELDU (NoELDU) \\
\hline & Number of cull cows culled today (NumberCulled) \\
\hline & Use of Salmonella vaccine (SalmonellaVaccine) \\
\hline & Sampling Season \\
\hline
\end{tabular}




\section{Table 3(on next page)}

Resistance phenotypes detected in Enterococcus spp. isolates. 


\begin{tabular}{|c|c|c|c|}
\hline Resistance Phenotypes observed in Enterococcus spp. (Antimicrobial class) & $\begin{array}{l}\text { Number of Cows } \\
\text { (Total } N=157 \text { ) }\end{array}$ & $\begin{array}{c}\text { The proportion } \\
\text { of cows (\%) }\end{array}$ & $95 \% \mathrm{CI}$ \\
\hline Nitrofuran antibacterial & 17 & 10.83 & $5.967-15.689$ \\
\hline Macrolides & 15 & 9.55 & $4.956-14.152$ \\
\hline Nitrofuran antibacterial, Macrolides & 15 & 9.55 & $4.956-14.152$ \\
\hline Oxazolidinones, Nitrofuran antibacterial, Macrolides ${ }^{\dagger}$ & 9 & 5.73 & 2.096-9.369 \\
\hline Oxazolidinones, Nitrofuran antibacterial & 8 & 5.1 & $1.656-8.535$ \\
\hline Tetracyclines & 7 & 4.46 & $1.23-7.687$ \\
\hline Oxazolidinones & 6 & 3.82 & $0.823-6.821$ \\
\hline Tetracyclines, Nitrofuran antibacterial & 6 & 3.82 & $0.823-6.821$ \\
\hline Tetracyclines, Nitrofuran antibacterial, Macrolides ${ }^{\dagger}$ & 5 & 3.18 & $0.438-5.931$ \\
\hline Streptogramin, Oxazolidinones, Nitrofuran antibacterial, Macrolides ${ }^{\dagger}$ & 4 & 2.55 & $0.083-5.013$ \\
\hline Tetracyclines, Amphenicols, Oxazolidinones, Nitrofuran antibacterial ${ }^{\dagger}$ & 4 & 2.55 & $0.083-5.013$ \\
\hline Streptogramin, Nitrofuran antibacterial & 4 & 2.55 & $0.083-5.013$ \\
\hline Tetracyclines, Amphenicols, Nitrofuran antibacterial, Macrolides ${ }^{\dagger}$ & 3 & 1.91 & $0.0-4.052$ \\
\hline Amphenicols, Oxazolidinones, Nitrofuran antibacterial, Macrolides ${ }^{\dagger}$ & 3 & 1.91 & $0.0-4.052$ \\
\hline Tetracyclines, Macrolides & 3 & 1.91 & $0.0-4.052$ \\
\hline
\end{tabular}




\begin{tabular}{|c|c|c|c|}
\hline Amphenicols, Oxazolidinones, Nitrofuran antibacterial $^{+}$ & 3 & 1.91 & $0.0-4.052$ \\
\hline Oxazolidinones, Macrolides & 3 & 1.91 & $0.0-4.052$ \\
\hline Amphenicols, Streptogramin, Oxazolidinones, Nitrofuran antibacterial, Macrolides ${ }^{\dagger}$ & 3 & 1.91 & $0.0-4.052$ \\
\hline Streptogramin & 2 & 1.27 & $0.0-3.028$ \\
\hline Tetracyclines, Oxazolidinones & 2 & 1.27 & $0.0-3.028$ \\
\hline Tetracyclines, Amphenicols, Nitrofuran antibacterial ${ }^{\dagger}$ & 2 & 1.27 & $0.0-3.028$ \\
\hline \multicolumn{4}{|l|}{ Tetracyclines, Amphenicols, Macrolides, Streptogramin, Oxazolidinones, } \\
\hline Nitrofuran antibacterial, Macrolides ${ }^{\dagger}$ & 2 & 1.27 & $0.0-3.028$ \\
\hline Amphenicols, Streptogramin, Oxazolidinones, Nitrofuran antibacterial ${ }^{+}$ & 2 & 1.27 & $0.0-3.028$ \\
\hline Tetracyclines, Oxazolidinones, Nitrofuran antibacterial, Macrolides ${ }^{\dagger}$ & 2 & 1.27 & $0.0-3.028$ \\
\hline Other single isolates of MDR phenotypes** & 23 & 14.72 & $9.118-20.180$ \\
\hline Other single isolates of AMR phenotypes* & 4 & 2.55 & $0.083-5.013$ \\
\hline
\end{tabular}

1 tRepresents phenotypes that are multi-drug resistant. **Other single isolates of MDR phenotypes from Enterococcus spp. isolates: 1)

2 tetracyclines, amphenicols, oxazolidinones, nitrofuran antibacterial, macrolides 2) oxazolidinones, nitrofuran antibacterial, macrolides,

3 glycopeptides 3) tetracyclines, amphenicols, oxazolidinones, macrolides 4) streptogramin, oxazolidinones, nitrofuran antibacterial 5) tetracyclines,

4 streptogramin, nitrofuran antibacterial, macrolides 6) tetracyclines, amphenicols, streptogramin, oxazolidinones, nitrofuran antibacterial,

5 macrolides 7) tetracyclines, amphenicols, macrolides, streptogramin, oxazolidinones, macrolides 8) amphenicols, macrolides, streptogramin, 
6 oxazolidinones, nitrofuran antibacterial 9) macrolides, streptogramin, oxazolidinones, nitrofuran antibacterials, macrolides 10) amphenicols,

7 nitrofuran antibacterial, macrolides 11) oxazolidinones, nitrofuran antibacterial, glycopeptides 12) amphenicols, oxazolidinones, macrolides 13)

8 tetracyclines, macrolides, oxazolidinones, nitrofuran antibacterial, macrolides 14) tetracyclines, streptogramin, nitrofuran antibacterial 15)

9 tetracyclines, macrolides, nitrofuran antibacterial, macrolides 16) amphenicols, streptogramin, macrolides 17) tetracyclines, streptogramin,

10 oxazolidinones 18) streptogramin, nitrofuran antibacterial, macrolides 19) tetracyclines, macrolides, oxazolidinones, nitrofuran antibacterial 20)

11 tetracyclines, oxazolidinones, nitrofuran antibacterial 21) tetracyclines, amphenicols, macrolides, oxazolidinones, nitrofuran antibacterial 22)

12 tetracyclines, amphenicols, macrolides, nitrofuran antibacterial 23) amphenicols, macrolides, nitrofuran antibacterial. *other single isolates of amr

13 phenotypes from enterococcus sp. isolates: 1) amphenicols, nitrofuran antibacterial 2) streptogramin, oxazolidinones 3) amphenicols, macrolides

14 4) macrolides, oxazolidinone 
Table 4 (on next page)

Resistant phenotypes detected in E. coli isolates. 


\begin{tabular}{|c|c|c|c|}
\hline Resistant Phenotypes observed in $E$. coli (Antimicrobial class) & $\begin{array}{l}\text { Number of Cows } \\
\text { (Total } N=81)\end{array}$ & $\begin{array}{l}\text { The proportion } \\
\text { of cows }(\%)\end{array}$ & 95\%CI \\
\hline Tetracyclines & 25 & 30.86 & $20.805-40.924$ \\
\hline Aminoglycosides & 11 & 13.58 & $6.12-21.041$ \\
\hline Cephalosporins & 9 & 11.11 & $4.267-17.955$ \\
\hline Aminoglycosides, Tetracyclines & 7 & 8.64 & $2.523-14.761$ \\
\hline Folate pathway antagonist & 5 & 6.17 & $0.932-11.414$ \\
\hline Amphenicols & 5 & 6.17 & $0.932-11.414$ \\
\hline Tetracyclines, Cephalosporins & 2 & 2.47 & $0.0-5.849$ \\
\hline Aminoglycosides, Tetracyclines, Amphenicols ${ }^{\dagger}$ & 2 & 2.47 & $0.0-5.849$ \\
\hline Other single isolates of AMR phenotypes* & 5 & 6.17 & $0.932-11.374$ \\
\hline Other single isolates of MDR phenotypes** & 5 & 6.17 & $0.932-11.414$ \\
\hline
\end{tabular}

2 tetracyclines 3) tetracyclines, folate pathway antagonist 6) cephalosporins, fluoroquinolones 7) amphenicols, folate pathway antagonist. ** other single isolates

3 of mdr phenotypes from e. coli: 1) amphenicols, folate pathway antagonist, aminoglycosides 2) amphenicols, tetracyclines, cephalosporins, aminoglycosides 3)

4 amphenicols, tetracyclines, folate pathway antagonist 4) amphenicols, tetracyclines, folate pathway antagonist, aminoglycosides 5) tetracyclines, cephalosporins,

5 fluoroquinolones, quinolones, aminoglycosides 


\section{Table 5 (on next page)}

Tetracycline antimicrobial resistance phenotype shared (highlighted in grey) between commensal bacteria (Enterococcus spp., E. coli) and Salmonella.

Each row represents bacterial resistance phenotypes of bacterial isolates from a single culled dairy cow. 


\begin{tabular}{|c|c|c|c|c|}
\hline \multicolumn{5}{|c|}{ Resistance Phenotypes observed } \\
\hline Salmonella & Enterococcus spp. & E. coli & Herd ID & Resistance \\
\hline Tetracyclines & Nitrofuran antibacterial & Aminoglycosides, Tetracyclines & 4 & AMR \\
\hline Tetracyclines & $\begin{array}{l}\text { Macrolides, Oxazolidinones, Nitrofuran } \\
\text { antibacterial }^{\dagger}\end{array}$ & Tetracyclines, Amphenicols & 4 & MDR \\
\hline Tetracyclines, Penicillins & Macrolides, Nitrofuran antibacterial & $\begin{array}{l}\text { Aminoglycosides, Tetracyclines, Folate } \\
\text { pathway antagonist, Amphenicols }^{\dagger}\end{array}$ & 4 & MDR \\
\hline Tetracyclines, Folate & Macrolides, Amphenicols & Tetracyclines & 4 & AMR \\
\hline pathway antagonist & & & & \\
\hline Tetracyclines & Tetracyclines, Nitrofuran antibacterial & $\begin{array}{l}\text { Tetracyclines, Folate pathway antagonist, } \\
\text { Amphenicols }^{\dagger}\end{array}$ & 4 & MDR \\
\hline Tetracyclines & Streptogramin, Oxazolidinones & Tetracyclines & 6 & AMR \\
\hline
\end{tabular}

†Represents phenotypes that are multi-drug phenotypes 